\title{
Mutations in the Glucose-6-Phosphatase Gene Are Associated with Glycogen Storage Disease Types 1a and 1aSP but Not 1b and 1c
}

\author{
Ke-Jian Lei," Leslie L. Shelly," Baochuan Lin," James B. Sidbury, ${ }^{\star}$ Yuan-Tsong Chen," Robert C. Nordlie, \\ and Janice Yang Chou* \\ *Human Genetics Branch, National Institute of Child Health and Human Development, National Institutes of Health, Bethesda, \\ Maryland 20892; ${ }^{\ddagger}$ Department of Pediatrics, Duke University Medical Center, Durham, North Carolina 27710; and ${ }^{8}$ Department of \\ Biochemistry and Molecular Biology, University of North Dakota School of Medicine, Grand Forks, North Dakota 58202
}

\begin{abstract}
Glycogen storage disease (GSD) type 1 , which is caused by the deficiency of glucose-6-phosphatase (G6Pase), is an autosomal recessive disease with heterogenous symptoms. Two models of G6Pase catalysis have been proposed to explain the observed heterogeneities. The translocase-catalytic unit model proposes that five GSD type 1 subgroups exist which correspond to defects in the G6Pase catalytic unit (1a), a stabilizing protein (1aSP), the glucose-6-P (1b), phosphate/ pyrophosphate (1c), and glucose (1d) translocases. Conversely, the conformation-substrate-transport model suggests that G6Pase is a single multifunctional membrane channel protein possessing both catalytic and substrate (or product) transport activities. We have recently demonstrated that mutations in the G6Pase catalytic unit cause GSD type 1a. To elucidate whether mutations in the G6Pase gene are responsible for other GSD type 1 subgroups, we characterized the G6Pase gene of GSD type 1b, 1c, and 1aSP patients. Our results show that the G6Pase gene of GSD type $1 \mathrm{~b}$ and 1c patients is normal, consistent with the translocasecatalytic unit model of G6Pase catalysis. However, a mutation in exon 2 that converts an Arg at codon 83 to a Cys (R83C) was identified in both G6Pase alleles of the type laSP patient. The $\mathrm{R83C}$ mutation was also demonstrated in one homozygous and five heterogenous GSD type 1a patients, indicating that type 1aSP is a misclassification of GSD type 1a. We have also analyzed the G6Pase gene of seven additional type 1a patients and uncovered two new mutations that cause GSD type 1a. (J. Clin. Invest. 1995. 95:234240.) Key words: type 1 glycogen storage disease - glucose-6phosphatase - genetic mutation - models of G6Pase catalysis
\end{abstract}

\section{Introduction}

Glycogen storage disease (GSD) $)^{1}$ type 1 is an inborn error of metabolism caused by the deficiency of glucose-6-phosphatase (G6Pase), the key enzyme in the homeostatic regulation of

Address correspondence to Janice Yang Chou, Building 10, Room 9S242, NIH, Bethesda, MD 20892. Phone: 301-496-1094; FAX: 301402-0234.

Received for publication 27 June 1994 and in revised form 15 September 1994.

1. Abbreviations used in this paper: GSD, glycogen storage disease; G6Pase, glucose-6-phosphatase.

The Journal of Clinical Investigation, Inc.

Volume 95, January 1995, 234-240 blood glucose concentrations $(1-4)$. It is an autosomal recessive disorder with an incidence of $\sim 1$ in 100,000 . The disease presents with clinical manifestations of severe hypoglycemia, hepatomegaly, growth retardation, lactic acidemia, hyperlipidemia, and hyperuricemia $(1,2)$. GSD type 1 has been classified into five subgroups, 1a, 1aSP, 1b, 1c, and 1d based upon observed biochemical and clinical heterogeneities (1, 2, 5-9). GSD type 1aSP is clinically indistinguishable from type 1a and was proposed to be caused by a defect in a $21-\mathrm{kD}$ stabilizing protein, SP, purified on the basis of its ability to stabilize the G6Pase catalytic unit in vitro $(5,10)$. Only one patient has been diagnosed with the type 1aSP disorder (5). G6Pase activity is very low or non-detectable in liver biopsy samples from GSD type 1a and 1aSP patients, regardless of the assay conditions. GSD type 1b patients suffer, in addition to the clinical symptoms observed in type 1a, neutropenia, neutrophil dysfunction, and recurrent bacterial infections $(11,12)$. Only a few cases of GSD type $1 \mathrm{c}$ have been reported and there is insufficient information for a general description of its clinical symptoms. Glucose-6-P or pyrophosphate hydrolytic activity in GSD type 1b and 1c patients, respectively, is totally or partially inactive in fresh liver biopsy specimens but becomes active after freezing and thawing or upon detergent treatment of the biopsy samples $(6,8)$.

To explain the heterogeneities observed among GSD type 1 patients, two models of G6Pase catalysis have been proposed. The multi-component translocase-catalytic unit model proposes that the five GSD subgroups correspond to defects in the G6Pase catalytic unit (1a), the putative SP (1aSP), the glucose-6-P (1b), phosphate/pyrophosphate (1c), and glucose (1d) translocases (5-9). This model also suggests that the G6Pase catalytic unit, situated on the lumen of the endoplasmic reticulum (ER), gains access to substrates in the cytosol by means of the associated translocases. The ability to restore enzyme activity in liver biopsy samples of type $1 \mathrm{~b}$ and 1c patients upon disruption of microsomes is consistent with the abolition of the translocation requirement. However, it is equally possible that activation of the enzyme after microsomal disruption could be caused by a conformational alteration of membrane-bound G6Pase, as proposed by the conformation-substrate-transport model (13). The latter suggests that G6Pase is a single multifunctional membrane channel protein possessing both catalytic and substrate (or product) transport activities.

Our laboratory has recently characterized the cDNA and gene encoding the catalytic unit of human G6Pase and identified, for the first time, several mutations in the G6Pase gene of GSD type 1a patients (14). Site-directed mutagenesis and transient expression assays demonstrated that these mutations abolish G6Pase activity, establishing the molecular basis of pathogenesis in GSD type 1a. In the present study, we characterized 
the G6Pase gene of GSD type 1b, 1c, and 1aSP patients in order to elucidate whether mutations in the G6Pase gene are responsible for other GSD type 1 subgroups. We also report additional mutations in the G6Pase gene of type la patients and the three most prevalent mutations that cause GSD type 1a.

\section{Methods}

Patients. We have analyzed the G6Pase gene of three GSD type 1b, one type 1c, the single type 1aSP, and seven type 1a patients. All patients present with clinical manifestations of hypoglycemia, hepatomegaly, and growth retardation. Three patients, MB, KF, and KZ, also have neutropenia and recurrent bacterial infections, and were positively diagnosed as GSD type $1 \mathrm{~b}$ by analyzing the glucose-6-P hydrolytic activity in liver biopsy samples. The activity was absent or partially present in fresh liver biopsy specimens but became active after freezing and thawing or upon detergent treatment of the biopsy samples. The defect in type $1 \mathrm{~b}$ patient MB has been extensively characterized $(6,11)$. The type 1c patient JJ, whose liver biopsy sample exhibited normal pyrophosphate hydrolytic activity upon detergent treatment, was reported by Nordlie et al. (8). The single type laSP patient, RP, was diagnosed and reported by Burchell and Waddell (5). The type 1aSP and the seven type 1a patients were diagnosed by the lack of G6Pase activity in liver biopsy samples, regardless of assay conditions.

Genomic DNA preparations from type 1a patient ML (GM00574) (submitted by Dr. R. Stevenson, Greenwood Genetic Center, Greenwood, NC), type 1b patient MB (GM03719) (submitted by Dr. A. Beaudet, Baylor College of Medicine, Houston, TX) were isolated from lymphoblasts obtained from National Institute of General Medical Sciences (NIGMS) Human Genetic Mutant Cell Repository (Camden, NJ). Genomic DNA preparations from the other GSD type 1 patients and available family members were extracted from blood samples. Genomic DNA was isolated using a Nucleon II kit obtained from Scotlab (Strathclyde, Scotland). Peripheral-blood samples were obtained with the informed consent of the patients.

Analysis of the G6Pase gene by polymerase chain reaction and DNA sequencing. The G6Pase gene of all GSD type 1 patients and available family members was characterized by amplifying the coding regions of each of the 5 exons and the corresponding intron-exon junctions by polymerase chain reaction (PCR) using five pairs of oligonucleotide primers containing intronic, 5'-untranslated, or $3^{\prime}$-untranslated sequences of the human G6Pase gene (14). The amplified fragments, I (306 bp), II (191 bp), III (209 bp), IV (259 bp), and V (646 bp), were subcloned using a TA cloning kit obtained from Invitrogen (San Diego, CA) and five or more subclones of each exon were sequenced and compared to a normal G6Pase gene to identify any mutations.

Construction of G6Pase mutants. The phG6Pase-1 cDNA (G6PaseWT) containing nucleotides 77 to 1156 of the entire coding region of the human G6Pase cDNA (14) was used as a template for mutant construction by PCR. The outside primers used in all constructs were nucleotides 77 to 96 (5'-AGGATGGAGGAAGGAATGAA-3', sense) and nucleotides 1153 to 1130 (5'-TTACAACGACTTCTTGTGCGGCTG-3', antisense). The two inside primers (nucleotides 1048 to 1074) for the $\Delta$ F327 mutant are 5'-CGTCTTGTCCTGCAAGAGTGCGGT(sense) and 5'-ACCGCACTCTTGCAGGACAAGACG (antisense) and the two inside primers (nucleotides 731 to 754 ) for mutant G222R are 5'-AGCTTCGCCATCCGATTTTATCTG-3' (sense) and 5'-CAGATAAAATCGGATGGCGAAGCT-3' (antisense). The outside primers contain an additional $\mathrm{XhoI}$ or $\mathrm{XbaI}$ linker. The amplified fragments were digested with XhoI and XbaI and ligated into a pSVL vector (Pharmacia Fine Chemicals, Piscataway, NJ). The sequence of all constructs was confirmed by DNA sequencing.

Expression in COS-1 cells and Northern-blot hybridization analysis. COS- 1 cells were grown at $37^{\circ} \mathrm{C}$ in Hepes-buffered Dulbecco's minimal essential medium supplemented with streptomycin, penicillin, and $4 \%$ fetal bovine serum. The WT or mutant G6Pase cDNA in a pSVL vector was transfected into COS-1 cells by the DEAE-dextran/chloroquine method (16). Mock transfections of COS-1 cells with the pSVL vector alone were used as controls. After incubation at $37^{\circ} \mathrm{C}$ for $3 \mathrm{~d}$, the transfected cultures were harvested for G6Pase assays or lysed for RNA isolation.

RNA was isolated by the guanidinium thiocyanate/ $\mathrm{CsCl}$ method (17), separated by electrophoresis in $1.2 \%$ agarose gels containing 2.2 $M$ formaldehyde, and transferred to Nytran membranes. The filters were hybridized at $42^{\circ} \mathrm{C}$ in the presence of the phG6Pase-1 probe as previously described (14).

Phosphohydrolase assay. Phosphohydrolase activity was determined essentially as described by Burchell et al. (18). Reaction mixtures (100 $\mu \mathrm{l})$ contained $50 \mathrm{mM}$ cacodylate buffer, $\mathrm{pH} 6.5,10 \mathrm{mM}$ glucose-6-P, $2 \mathrm{mM}$ EDTA, and appropriate amounts of cell homogenates and were incubated at $30^{\circ} \mathrm{C}$ for $10 \mathrm{~min}$. Absorbance of each sample was determined at $820 \mathrm{~nm}$ and is related to the amount of phosphate released using a standard curve constructed by a stock of inorganic phosphate solution. Results are present as the mean $\pm \mathrm{SD}$.

\section{Results}

The deduced amino acid sequence of the G6Pase catalytic unit of GSD type $1 b$ and $1 c$ patients is normal. To test the two proposed models of G6Pase catalysis, we have undertaken studies to invoke or eliminate for consideration defects in the G6Pase gene as a factor in GSD type $1 \mathrm{~b}, 1 \mathrm{c}$, or $1 \mathrm{aSP}$. Biochemically, the glucose-6-P or pyrophosphate hydrolytic activity in
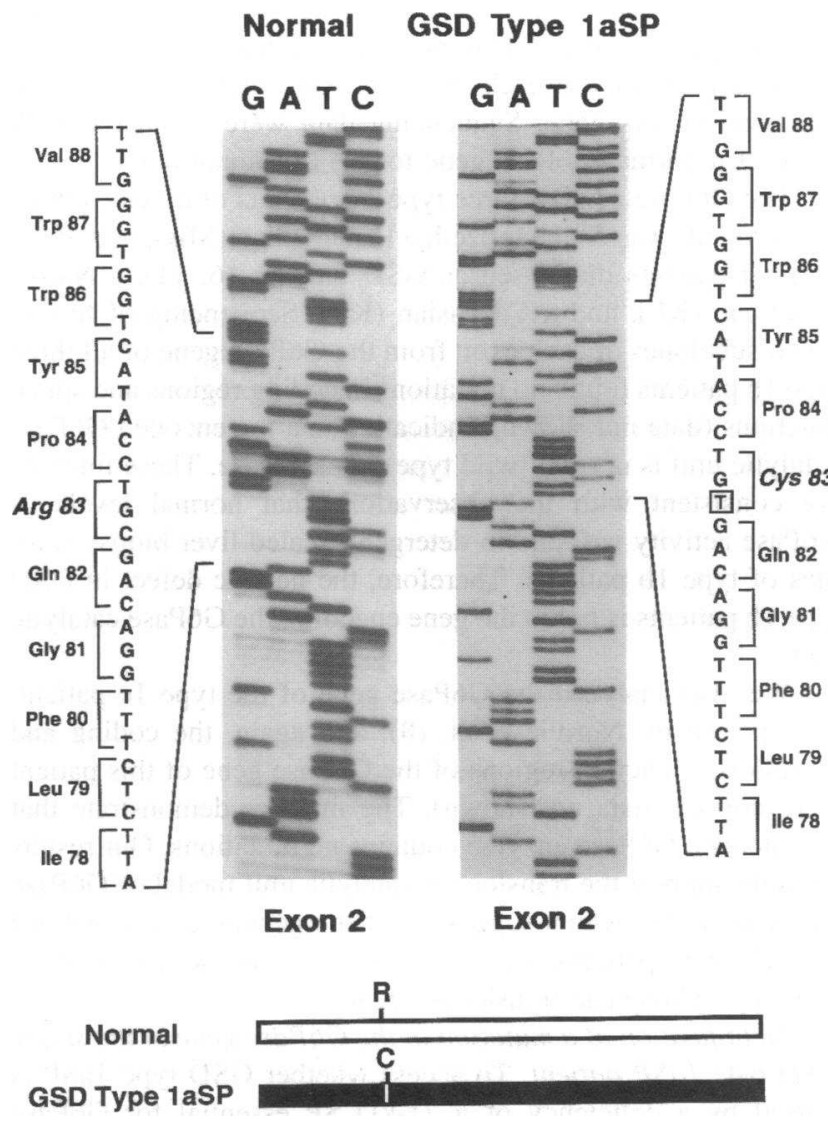

Figure 1. Autoradiograms of Sanger nucleotide sequencing reactions of the G6Pase gene from normal and the GSD type laSP patient. This type laSP patient (RP) contains a $\mathrm{C}$ to $\mathrm{T}$ mutation (boxed) at nucleotide 326 (exon 2) converting an Arg at codon 83 to a Cys (R83C) in both G6Pase alleles. 
Table I. Mutations in the G6Pase Genes of GSD type la Patients

\begin{tabular}{|c|c|c|c|}
\hline GSD Type 1a & Mutation 1 & Mutation 2 & Genotype \\
\hline \multicolumn{4}{|c|}{ nucleotide/amino acid } \\
\hline AN & C326T/R83C (5/5) & & Homozygote \\
\hline AN, father & C326T/R83C (2/8) & & \\
\hline AN, mother & C326T/R83C (2/6) & & \\
\hline LT & C1118T/Q347X (5/5) & & Homozygote \\
\hline LT, mother & C1118T/Q347X (5/7) & & \\
\hline $\mathrm{CA}$ & C326T/R83C (4/9) & 1057delTTC/ $\Delta \mathrm{F} 327(5 / 7)$ & Heterozygote \\
\hline CA, mother & C326T/R83C (2/6) & & \\
\hline JD & C326T/R83C (2/5) & C1118T/Q347X (4/6) & Heterozygote \\
\hline JD, father & C326T/R83C (3/5) & & \\
\hline JD, mother & & C118T/Q347X (2/5) & \\
\hline PG & C326T/R83C (2/6) & 459insTA/130X (4/6) & Heterozygote \\
\hline PG, father & C326T/R83C (4/6) & & \\
\hline PG, mother & & 459insTA/130X (2/5) & \\
\hline RH & 459insTA/130X (4/6) & C1118T/Q347X (4/7) & Heterozygote \\
\hline RH, mother & 459insTA/130X (7/9) & & \\
\hline ML & G743C/G222R 3/6) & C1118T/Q347X (3/6) & Heterozygote \\
\hline
\end{tabular}

The liver biopsy specimens of these patients exhibited very low or nondetectable G6Pase activity, regardless of assay conditions. Numbers in parentheses are numbers of subclones that contained the mutation versus the numbers of subclones analyzed.

type $1 \mathrm{~b}$ and $1 \mathrm{c}$ patients becomes active in detergent-treated liver biopsy samples $(6,8)$. To determine the status of the G6Pase gene in type $1 \mathrm{~b}$ and $1 \mathrm{c}$ patients, we amplified and sequenced the coding regions of each of the 5 exons and all intron-exon junctions of this gene. Sequencing data were compared with those of a normal G6Pase gene to identify mutations.

The G6Pase gene of three type $1 \mathrm{~b}$ patients of diverse ethnic backgrounds was characterized: a Panamanian (MB), one of the original patients diagnosed as GSD type $1 b(6,11)$, a Native American (KF), and a Caucasian (KZ). Sequencing of five to seven subclones of each exon from the G6Pase gene of all three type $1 \mathrm{~b}$ patients found no mutations in coding regions and splice junctions (data not shown), indicating that the encoded G6Pase catalytic unit is normal (wild type) in each case. These findings are consistent with the observations that normal levels of G6Pase activity is found in detergent-treated liver biopsy samples of type $1 \mathrm{~b}$ patients. Therefore, the genetic defect in GSD type $1 \mathrm{~b}$ patients is not in the gene encoding the G6Pase catalytic unit.

We also analyzed the G6Pase gene of the type 1c patient, $\mathrm{JJ}$, reported by Nordlie et al. (8), and again, the coding and intron/exon junction regions of the G6Pase gene of this patient were normal (data not shown). The findings demonstrate that the encoded G6Pase enzyme contains no mutations. Our results strongly support the translocase-catalytic unit model of G6Pase catalysis (6-9), which suggests that the genetic defect in GSD type $1 \mathrm{~b}$ or $1 \mathrm{c}$ patients is in the associated glucose-6-P or phosphate/pyrophosphate translocase gene.

Identification of a mutation in the G6Pase gene of the single GSD type laSP patient. To access whether GSD type 1aSP is caused by a deficiency of a 21-kD SP essential for G6Pase activity but otherwise normal G6Pase $(5,9,10)$, we examined the G6Pase gene from the single known patient, RP. It has been shown that type 1aSP is clinically indistinguishable from type 1a, suggesting that the symptoms observed in this type laSP patient could be caused by mutations in the G6Pase gene, simi- lar to type 1a patients. Sequence analysis indicated that the sequences of exons $1,3,4$, and 5 and all intron/exon junctions in the G6Pase gene of the type laSP patient were normal. However, exon 2 contained a $\mathrm{C}$ to $\mathrm{T}$ transition at nucleotide 326 converting an Arg at codon 83 to a Cys (R83C) (Fig. 1). All five subclones contained this mutation, indicating that RP is homozygous for the R83C mutation. As GSD type 1 is an autosomal recessive disorder, it is predicted that both parents would be heterozygous for the mutation. This was confirmed by sequencing the G6Pase gene of both parents. As expected, three of the six exon 2 subclones of the G6Pase gene of the mother and two of the six exon 2 subclones of the G6Pase gene of the father had the $\mathrm{C}$ to $\mathrm{T}$ mutation at nucleotide 326 converting an Arg-83 to a Cys (data not shown). The sister of patient RP, clinically normal, contained two normal G6Pase alleles (data not shown).

Identification of the R83C mutation in the G6Pase gene of a homozygous GSD type la patient. The R83C mutation has been previously identified in two GSD type 1a patients; both were compound heterozygotes $(14,15)$. To determine if the type 1aSP phenotype corresponds to patients homozygous for the R83C mutation, we screened additional type 1a patients for this mutation. Sequence analysis indicated that a type 1a patient, AN, also carried the R83C mutation in exon 2 of both G6Pase alleles (Table I). The sequences of exons 1, 3, 4, and 5 of the gene of patient AN were normal. Moreover, both parents of AN were heterozygous for the R83C mutation (Table I).

Identification of mutations in the G6Pase gene of six additional GSD type la patients. To understand further the molecular basis of the GSD type 1a disorder, we characterized, in addition to patient AN, the G6Pase gene of six additional GSD type 1a patients. Among one homozygous and five heterozygous type la patients, five different mutations at the G6Pase locus have been identified (Table I). Three of the mutations, R83C, 459insTA (insertion of TA after nucleotide 459 that yields a truncated G6Pase of 129 residues), and Q347X (a C to T transi- 


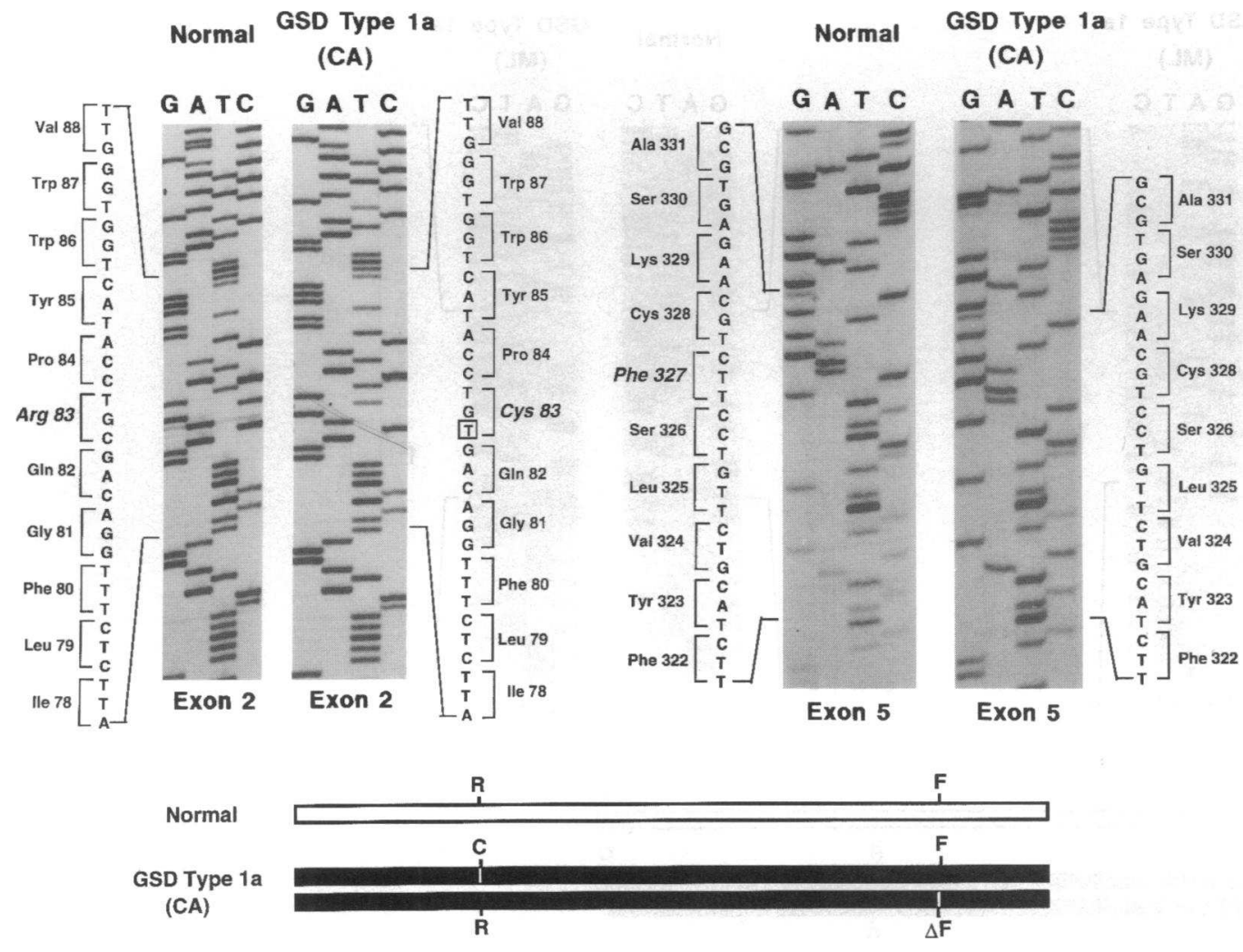

Figure 2. Autoradiograms of Sanger nucleotide sequencing reactions of the G6Pase gene from normal and GSD type 1a patient CA. Patient CA contains a C to T (boxed) mutation at nucleotide 326 in exon 2 that converts Arg-83 to a Cys (R83C) and a codon (TTC, nucleotides 1058 to $1060)$ deletion for Phe-327 ( $\Delta \mathrm{F} 327)$ in exon 5.

tion at nucleotide 1118 that converts Gln-347 to a stop codon) have been previously described $(14,15)$. Two new mutations, $\triangle F 327$ and G222R, were identified in patients CA and ML, respectively. The genetic heritage of the patients was identified by the ratio of mutation-containing exon subclones to total subclones analyzed. A ratio of one indicates the patient is homozygous. This was further confirmed by sequence analysis of the G6Pase gene of the available family members (Table I).

Patient CA is a compound heterozygote; two different mutations were detected in the G6Pase gene of this patient (Fig. 2). The mutations are R83C and a codon (TTC) deletion for Phe327 (nucleotides 1058 to 1060) in exon 5 (Fig. 2). Patient ML is also a compound heterozygote containing two different mutations in exon 5 at the G6Pase locus: a $\mathrm{G}$ to $\mathrm{C}$ transversion at nucleotide 743 that converts a Gly at codon 222 to an Arg (G222R) and a $\mathrm{C}$ to $\mathrm{T}$ transition at nucleotide 1118 that converts a Gln at codon 347 to a stop codon (Q347X) (Fig. 3).

We have previously characterized the G6Pase gene of four unrelated GSD type 1a patients and uncovered four different mutations, R83C, Q347X, 459insTA, and R295C (a C to T mutation at nucleotide 962 that converts Arg-295 to a Cys) (14, 15). In summary, we have analyzed the G6Pase gene of one type 1aSP and eleven unrelated type 1a patients and uncovered six different mutations. The relative incidence of the mutations in the G6Pase alleles are shown in Fig. 4. R83C, Q347X, and 459insTA, which constitute $87 \%$ of the observed mutations, may represent the most prevalent mutations found in GSD type 1a.

Characterization of G6Pase- $\Delta F 327$ and G6Pase-G222R mutants. The liver biopsy specimens of patients RP, AN, LT, CA, JD, PG, RH, and ML had very low or undetectable phosphohydrolase activity, suggesting that mutations identified in the G6Pase gene of these patients inactivated the G6Pase enzyme. In earlier studies, we showed that the R83C, R295C, or Q347X mutant G6Pase had no detectable phosphohydrolase activity $(14,15)$. To investigate the importance of amino acid residue $F 327$ and $G 222$, we constructed mutants that eliminated Phe-327 (G6Pase- $\Delta$ F327) or that changed Gly-222 to Arg (G6Pase-G222R) and analyzed phosphohydrolase activities after transient expression of WT or mutant G6Pase cDNA into COS-1 cells (Fig. 5 A). The G6Pase- $\Delta$ F327 mutant generated no enzyme activity. On the other hand, the G6Pase-G222R mutant G6Pase had a reproducibly low level of phosphohydrolase activity.

Northern-blot hybridization analysis of G6Pase transcripts from transfected cells showed that WT as well as $\Delta F 327$ and G222R mutant G6Pase mRNAs were expressed at similar levels (Fig. $5 B$ ), indicating that the reduction in enzyme activity was due to the defect in the G6Pase protein and not due to a decrease in transfection efficiency.

\section{Discussion}

In the present report, we have examined the G6Pase gene of GSD type $1 b$ and 1c patients and demonstrated that the coding regions and intron/exon junctions are all normal. This is consistent with the observations that, in contrast to the type 1a disorder, both type $1 \mathrm{~b}$ and $1 \mathrm{c}$ patients have normal G6Pase activity 


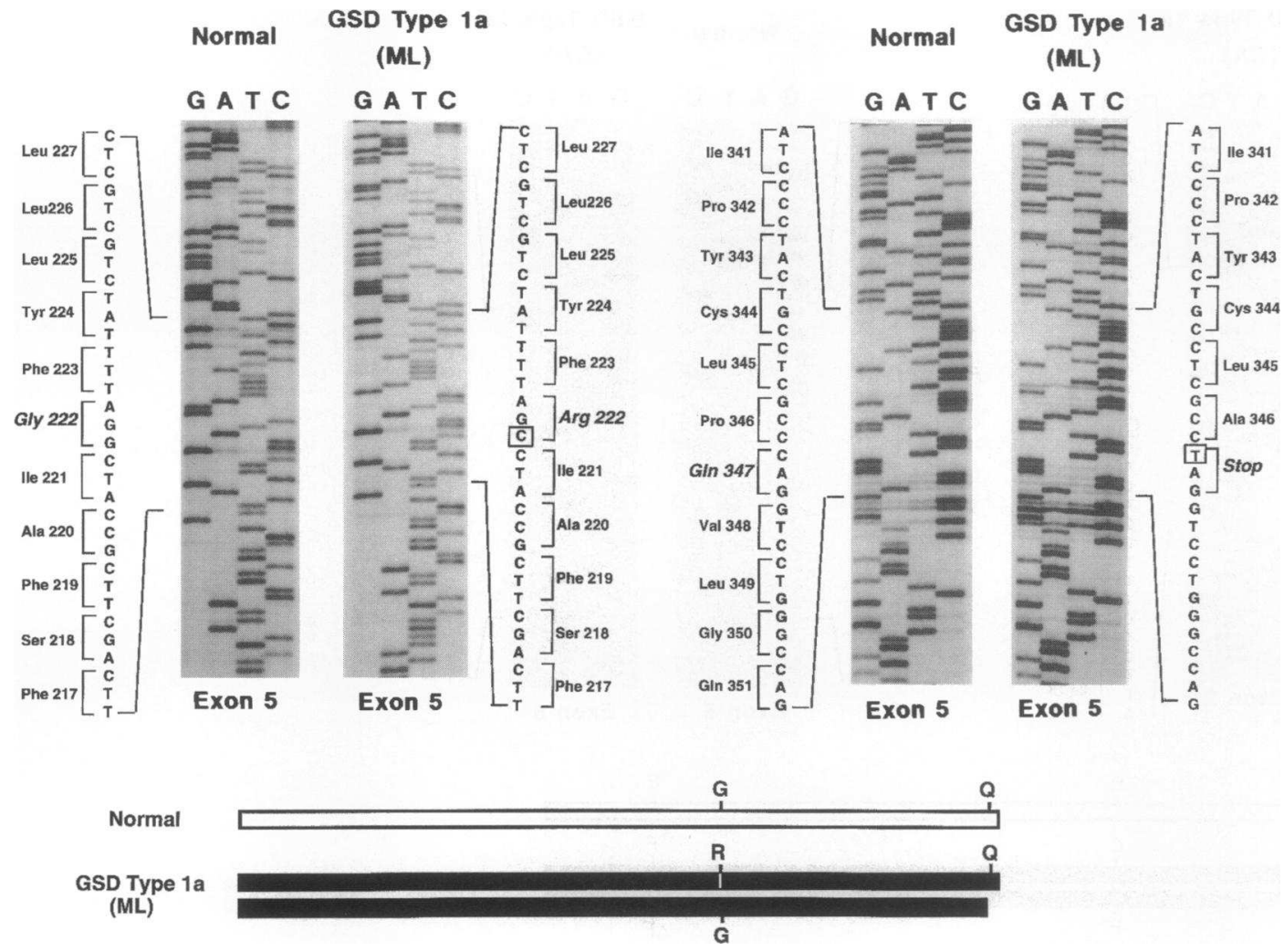

Figure 3. Autoradiograms of Sanger nucleotide sequencing reactions of the G6Pase gene from normal and GSD type 1a patient ML. Patient ML contains a G to C (boxed) mutation at nucleotide 743 in exon 5 that converts Gly-222 to an $\operatorname{Arg}(\mathrm{G} 222 \mathrm{R})$ and a $\mathrm{C}$ to $\mathrm{T}$ transition at nucleotide 1118 in exon 5 that converts Gln-347 to a stop codon (Q347X).

once the liver biopsy specimens are treated with detergent surmounting an apparent abnormal latency of an otherwise functional G6Pase enzyme in the type $1 b$ and $1 c$ patients $(6,8)$. Therefore, a search for a mutation outside the coding region of the G6Pase gene is now unwarranted. Both the clinical and biochemical heterogeneities observed in the GSD type 1 disorder support the multi-component nature of the G6Pase system as proposed by the translocase-catalytic unit model of G6Pase catalysis (6-9). It is now established that at least two or more loci control the activity of glucose-6-P hydrolysis. The genetic defect(s) in type $1 \mathrm{~b}$ and $1 \mathrm{c}$ patients, also phenotypically deficient in G6Pase activity, remains to be elucidated. Biochemical analysis of G6Pase activity in liver biopsy samples of type $1 \mathrm{~b}$ and $1 \mathrm{c}$ patients has attributed the defects to the putative translocases essential for G6Pase activity in the in vivo conditions $(6,8)$. The clinical manifestations of type $1 \mathrm{~b}$ and $1 \mathrm{c}$ patients appear to be as severe as that of the 1a patients, arguing that some other component of the G6Pase complex is equally important to the hydrolysis of glucose-6-P.

G6Pase is known to be an extremely unstable protein losing enzyme activity during conventional purification procedures (3, 4). A $21-\mathrm{kD}$ polypeptide has been isolated by Burchell et al. (10) that is reported to be closely associated with the G6Pase catalytic unit and responsible for stabilization of the enzyme. Screening of $>400$ human liver biopsy samples, including those from GSD type 1 patients, with monospecific antibody to SP detected one specimen from patient RP that lacked SP (5, 9). On this basis, a new subgroup, designated GSD type 1aSP, was established. In this report, we demonstrate that the type
laSP patient, RP, is homozygous for $\mathbf{R} 83 \mathrm{C}$ mutation in the G6Pase enzyme. The same mutation has been detected in one other homozygous and five compound heterozygous type 1a patients, demonstrating that the homozygous R83C mutation is not unique to the type "laSP" phenotype.

Several lines of evidence indicate that the G6Pase catalytic unit is enzymatically active in the absence of the putative SP and that GSD type 1aSP, like type 1a, is caused exclusively by mutations that inactivate the human G6Pase. First, the ability of the SP to stabilize G6Pase was not unequivocally demonstrated and the SP may in fact be a ferritin subunit (19). The diagnosis of the type 1aSP phenotype was based solely on immunoblots which showed that the liver biopsy sample of patient $R P$ did not react with an antiserum raised against the putative SP $(5,9)$. This patient lacked an SP which has not been demonstrated to be required for G6Pase activity. Second, rat G6Pase was recently purified to near homogeneity and demonstrated to be a single polypeptide of $35 \mathrm{kD}$ (20). The purified G6Pase is enzymatically active in the absence of any associated SP. Third, the loss of hepatic G6Pase activity of this patient has been unequivocally demonstrated in the present study to be the result of a mutation (R83C) in the G6Pase gene. Genetic analysis of DNA isolated from the parents of RP indicated that the R83C mutation was inherited in an autosomal recessive manner. The $\mathrm{C}$ to $\mathrm{T}$ transition in exon 2 (CGT to TGT) in the G6Pase gene occurs in a CpG doublet, a known hotspot for mutation due to potential methylation of the doublet (21).

Analysis of the G6Pase gene in eleven unrelated patients diagnosed as GSD type 1a and a single patient diagnosed as 


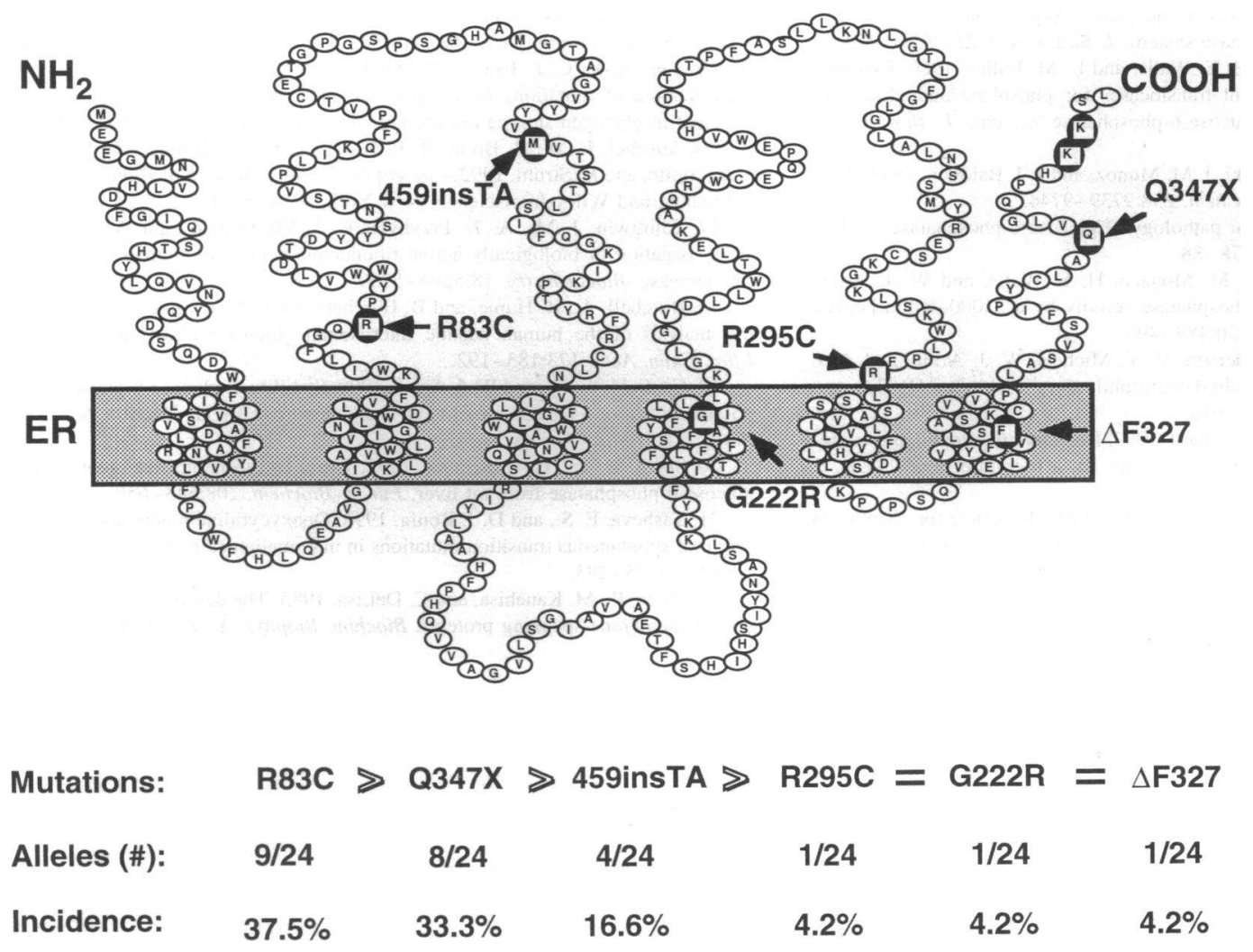

Figure 4. Location of mutations in G6Pase alleles. Transmembrane spanning domains were identified by the method of Klein et al. (22) using the PC/Gene Program. The predicted secondary structure of human G6Pase is depicted and the six mutations identified thus far are denoted by arrows. The incidence of mutant alleles in GSD type la patients characterized to date are listed below the diagram.

type "1aSP" have uncovered six independent mutations. The R83C (14), 459insTA (14), R295C (14), $\Delta$ F327, and Q347X (15) mutations abolished G6Pase catalytic activity completely whereas the G222R mutation greatly reduced it. The loss of enzyme activity in G6Pase lacking Phe-327, located in the sixth putative transmembrane segment of human G6Pase (Fig. 4), suggests that structural integrity of the membrane-spanning seg-

$\begin{array}{lr}\text { A } & \text { Phosphohydrolase Activity } \\ \text { Mock } & 11.7 \pm 2.4 \\ \text { WT } & 115.8 \pm 4.5 \\ \triangle \text { F327 } & 10.4 \pm 1.4 \\ \text { G222R } & 15.9 \pm 3.6\end{array}$

Figure 5. (A) Analysis of G6Pase phosphohydrolase activity and $(B)$ mRNA expression after transient expression of WT and mutant G6Pase cDNAs in COS-1 cells. Phosphohydrolase activity in whole homogenates was assayed in re-

B

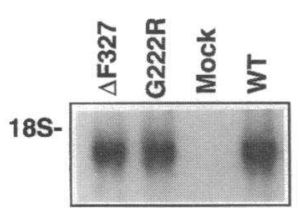
mM glucose-6-P using two independent isolates of each construct in three separate transfections. The activity is expressed as $\mathrm{nmol} / \mathrm{min}$ per $\mathrm{mg}$ pro- ment is important for G6Pase catalysis. In summary, our studies strongly support the translocase-catalytic unit model of G6Pase catalysis after simplifying the model by discarding one component, SP. In addition, these studies provide important information concerning the critical amino acids in G6Pase catalysis.

\section{Acknowledgments}

We thank Kerri Lamance, Dr. Arthur L Beaudet, and the other metabolic physicians at Baylor College of Medicine for providing patient samples, Ms. C.-J. Pan and J.-L. Liu for technical assistance, and Drs. Margaret Chamberlin, Ida Owens, Lawrence Charnas, and Anil Mukherjee for critical reading of the manuscript.

\section{References}

1. Hers, H.-G., F. Van Hoof, and T. de Barsy. 1989. Glycogen storage diseases In The Metabolic Basis of Inherited Disease. C. R. Scriver, A. L. Beaudet, R. Charles, W. S. Sly, and D. Valle, editors. McGraw-Hill Inc., New York. 425452 .

2. Beaudet, A. L. 1991. Harrison's Principles of Internal Medicine. 12th tein and data are presented as the mean \pm SD. After subtracting the background activity (mock), the $\Delta F 327$ mutant exhibited no detectable activity whereas the G222R mutant retained $\sim 4 \%$ of WT activity. edition. J. D. Wilson, E. Braunwald, K. J. Isselbacher, R. G. Petersdorf, J. B., Martin, A. S., Fauci, and R. K. Root, editors. McGraw-Hill Inc., New York. 1854-1860.

3. Nordlie, R. C., and K. A. Sukalski. 1985. Multifunctional glucose-6-phosphatase: a critical review. In The Enzymes of Biological Membranes. 2nd edition. A. N. Martonosi, editor. Plenum Press, New York. 349-398.

4. Sukalski, K. A., and R. C. Nordlie. 1989. Glucose-6-phosphatase: two concepts of membrane-function relationship. Adv. Enzymol. 62:93-117.

5. Burchell A., and I. D. Waddell. 1990. Diagnosis of a novel glycogen storage disease: type 1aSP. J. Inherited Metab. Dis. 13:247-249.

6. Lange, A. J., W. J. Arion, and A. L. Beaudet. 1980. Type 1 b glycogen 
storage disease is caused by a defect in the glucose-6-phosphate translocase of the microsomal glucose-6-phosphatase system. J. Biol. Chem. 255:8381-8384.

7. Arion, W. J., A. J. Lange, H. E. Walls, and L. M. Ballas. 1980. Evidence of the participation of independent translocases for phosphate and glucose-6phosphate in the microsomal glucose-6-phosphatase system. J. Biol. Chem. 255:10396-10406.

8. Nordlie, R. C., K. A. Sukalski, J. M. Munoz, and J. J. Baldwin. 1983. Type 1c, a novel glycogenosis. J. Biol. Chem. 258:9739-9744.

9. Burchell, A. 1990. Molecular pathology of glucose-6-phosphatase. FASEB (Fed. Am. Soc. Exp. Biol.) J. 4:2978-88.

10. Burchell, A., B. Burchell, M. Monaco, H. E. Walls, and W. J. Arion. 1985. Stabilization of glucose-6-phosphatase activity by a 21000 -dalton hepatic microsomal protein. Biochem. J. 230:489-495.

11. Beaudet, A. L., D. C. Anderson, V. V. Michels, W. J. Arion, and A. J. Lange. 1980. Neutropenia and impaired neutrophil migration in type 1B glycogen storage disease. J. Pediatr. 97:906-910.

12. Gitzemann, R., and N. U. Bosshard. 1993. Defective neutrophil and monocyte functions in glycogen storage disease type 1b: a literature review. Eur. J. Pediatr. 152(Suppl 1):S33-S38.

13. Schulze, H. U., B. Nolte, and R. Kannler. 1986. Evidence for changes in the conformational status of rat liver microsomal glucose-6-phosphate: phosphohydrolase during detergent-dependent membrane modification. J. Biol. Chem. 261:16571-16578.

14. Lei, K.-J., L. L. Shelly, C.-J. Pan, J. B. Sidbury, and J. Y. Chou. 1993.
Mutations in the glucose-6-phosphatase gene that cause glycogen storage disease type 1a. Science (Wash. DC). 262:580-583.

15. Lei, K.-J., C.-J. Pan, L. L. Shelly, J.-L. Liu, and J. Y. Chou. 1994 Identification of mutations in the gene for glucose-6-phosphatase, the enzyme deficient in glycogen storage disease type 1a. J. Clin. Invest. 93:1994-1999.

16. Ausubel, F. M., R. Brent, R. E. Kingston, D. D. Moore, J. G. Seidman, J. A., Smith, and K. Struhl. 1992. Current Protocols in Molecular Biology. Greene Publishing and Wiley-Interscience, New York. 9.2.1-9.2.6.

17. Chirgwin, J. M., A. E. Przybyla, R. J. MacDonald, and W. J. Rutter. 1979. Isolation of biologically active ribonucleic acid from sources enriched in ribonuclease. Biochemistry. 18:5294-5299.

18. Burchell, A., R. Hume, and B. Burchell. 1988. A new microtechnique for the analysis of the human hepatic microsomal glucose-6-phosphatase system. Clim. Chim. Acta. 173:183-192.

19. Canfield, W. K., and W. J. Arion. 1990. Stability of glucose-6-phosphatase: possible roles for a ferritin subunit and molybdenum. FASEB (Fed. Am. Soc. Exp. Biol.) J. 4:2121a. (Abstr.)

20. Speth, M., and H.-U. Schulze. 1992. The purification of a detergent-soluble glucose-6-phosphatase from rat liver. Eur. J. Biochem. 208:643-650.

21. Tasheva, E. S., and D. J. Roufa. 1993. Deoxycytidine methylation and the origin of spontaneous transition mutations in mammalian cells. Somatic Cell Mol. Genet. 19:275-283.

22. Klein, P., M. Kanehisa, and C. DeLisa. 1985. The detection and classification of membrane-spanning proteins. Biochim. Biophys. Acta. 815:468-476. 